\title{
Control of Ascaris infection by chemotherapy: which is the most cost-effective option?
}

H. L. Guyatt ${ }^{1}$, M. S. Chan ${ }^{2}$, G. F. Medley ${ }^{3}$ and D. A. P. Bundy ${ }^{2}{ }^{1}$ Department of Public Health and Epidemiology, Swiss Tropical Institute, Socinstrasse 57, CH-4002 Basel, Switzerland; ${ }^{2}$ Department of Zoology, University of Oxford, South Parks Road, Oxford, OX1 3PS, UK; ${ }^{3}$ Ecosystems Analysis and Management Group, Department of Biological Sciences, University of Warwick, Coventry, CV4 7AL, UK

\begin{abstract}
Cost-effectiveness analysis is used to predict the optimal design of mass chemotherapy strategies in controlling Ascaris lumbricoides infection. The question of who to treat, how many to treat, and how often to treat are addressed using a population dynamic model of helminth transmission that assesses effectiveness in terms of disease reduction, combined with cost data from an actual control programme. Child-targeted treatment can be more cost-effective than population treatmient in reducing the number of disease cases. The model also implies that, in the circumstances described here, enhancing coverage is a more cost-effective approach than increasing frequency of treatment.
\end{abstract}

Keywords: Ascaris, chemotherapy, cost-effectiveness

\section{Introduction}

Chemotherapy is advocated as an affordable and effective control measure for reducing intestinal nematode morbidity (SAVIOLI et al., 1992; WARREN et al., 1992). However, the most cost-effective strategy of delivering chemotherapy to endemic communities remains uncertain.

Theoretical analysis has shown that selective treatment (identifying and treating only those who are diagnosed as infected) can provide substantial benefits both in terms of reducing the abundance of the parasite and the number of treatments administered (ANDERSON \& MAY, 1982). However, the high cost of diagnosis and poor compliance result in selective treatment being more expensive and less effective than mass treatment in controlling intestinal nematode infection (PRESCOTT, 1987; BUNDY, 1990). However, if mass treatment is the option of choice, there are still other design aspects which have to be considered. One issue is the target population: at whom should the drug be targeted? - the whole population, only high-risk groups such as school-age children, or a combination of both? One also needs to consider how often the drug should be given and what proportion of the target population should be covered. It is not known, for instance, whether it is better to treat more people less frequently or less people more frequently. Ideally, one would require extensive field studies to investigate these issues. However, such studies are expensive and time-consuming, as well as producing data restricted to a given endemic locality.

A more immediate investigation into the most appropriate ways of delivering chemotherapy can be undertaken using theoretical analysis. A population dynamic model of helminth transmission has been developed by MEDLEY et al. (1993) which allows estimation of the effectiveness of different approaches to chemotherapy. This model has already been used to investigate how often mass treatment should be administered in order to maximize cost-effectiveness (GUYATT et al., 1993). This study showed that less frequent treatments were most cost-effective in reducing the number of cases with heavy Ascaris lumbricoides infections, and that measuring effectiveness in terms of only the number of infections prevented produced misleading results. This study was confined to population mass treatment, and assumed a fixed population coverage.

The model has now been refined to include separate age groups, which allows investigation into age-targeted approaches to control (CHAN et al., in press). Although child-targeted treatment will never be more effective

Address for correspondence: Dr Helen Guyatt, Department of Zoology, University of Oxford, South Parks Road, Oxford, OX1 3PS, UK. than population treatment, it is less costly and may prove to be more cost-effective since this target group has the highest intensities of infection, and is therefore most likely to suffer disease and to be responsible for a larger proportion of the transmission stages (WARREN et al., 1992). Indeed, the treatment of children only may also lead to the reduction of infection in untreated adults simply from a reduction in the number of transmission stages; this has been observed in field studies (BUNDY et al., 1990). The age-structured population dynamic model has been shown to be a good fit to the observed data by CHAN et al. (in press), who also showed that population treatment followed by child-targeted treatment was more effective than child-targeted treatment alone, though the costs of these 2 programmes were not compared.

In the first part of this paper, the cost-effectiveness of 3 options for chemotherapeutic control of $A$. lumbricoides in a high and low transmission arca arc comparcd: population treatment, child-targeted treatment, and population treatment followed by child-targeted treatment. In this example, frequency of treatment and coverage are fixed. The interaction between coverage and frequency of treatment is complex and non-linear, and it is not immediately clear whether it is better to treat more people less frequently or less people more frequently. The second part of this paper will address this issue by comparing the cost-effectiveness of different combinations of frequency and coverage in delivering child-targeted treatment in a high transmission area. The predictions from the analyses will be used to provide some guidelines to the most cost-effective approaches to $A$. lumbricoides control.

\section{Methods \\ The model}

The model used to assess effectiveness has been described in detail by CHAN et al. (in press). Briefly, the host population is divided into 2 groups, children and adults, both of which harbour a parasite population of mature worms. A further parasite population is conceptualized as a 'pool' of free-living infective stages in the environment. The host population can contaminate the environment by faeces containing eggs which develop into infective stages, and can become infected by contact with these infective stages. The 2 host groups differ in that the children both acquire infections and contaminate the environment at a higher rate than adults. The equilibrium worm population is set up using epidemiological parameter estimates of the life expectancy of the adult worm, the distribution of infection, and the rates of transmission. Left undisturbed, the parasite population will remain stable due to continual natural mortality of adult worms and infection by infective stages. Treatment 
is simulated as instantaneous killing of adult worms in treated individuals. The efficacy of the drug is expressed as a proportion, an efficacy of 1.0 resulting in all worms in treated individuals being killed. The coverage and frequency of treatment can be varied and treatment given to the entire population or to one group alone. The changes in mean worm burden, prevalence of infection and prevalence of morbidity in both host groups can be followed during and after several rounds of treatment. Morbidity is assumed to be associated with heavy infection by using a threshold worm burden at which morbidity occurs.

\section{Base case}

Treatment was assumed to be directed at $A$. lumbricoides in high and low transmission areas. The epidemiological parameters used in the model are given in Table 1. Population size was assumed to be 100000 , of whom

Table 1. The epidemiological parameters for Ascaris lumbricoides transmission in a high and low transmission area

\begin{tabular}{lcccc}
\hline & \multicolumn{4}{c}{ Transmission area } \\
Parameter $^{\mathbf{a}}$ & Children & Adults & Children & Adults \\
\hline$W^{\star}$ & 7 & 3 & 12 & 6 \\
$T$ & 20 & 20 & 20 & 20 \\
$k$ & 0.54 & 0.54 & 0.54 & 0.54 \\
$\mathrm{R}_{0}$ & 2.5 & 1.25 & 3 & 1.5 \\
$\mu$ (years) & 1 & 1 & 1 & 1 \\
\hline
\end{tabular}

aThe basic reproductive rate $\left(R_{0}\right)$ and equilibrium worm burden $\left(\mathrm{W}^{\star}\right)$ were based on estimates by CROLL et al. (1982) for the high transmission area and CHAI et al. (1985) and BUNDY et al. (1987) for the low transmission area. The parameter $k$, an index of the degree of parasite aggregation, was taken from GUYATT $e t$ al. $(1990)$ and the disease threshold (T) was based on analysis by GUYATT \& BUNDY (1991). The adult worms' life expectancy (u) was based on studies reported by MULLER (1975).

40000 were children ( $\leqslant 15$ years old) and 60000 were adults ( $>15$ years). Mass yearly treatment was assumed to be administered in years $1-5 ; 90 \%$ of children were covered by the programme, and $50 \%$ of adults, reflecting typical levels of age-dependent compliance with a high rate of school attendance. The drug efficacy was assumed to be 0.9 for both children and adults.

\section{Scenarios}

Two scenarios extended from the base case were investigated. The first was a comparison of 3 alternatives for a 5 -year mass annual treatment programme in high and low transmission areas. The 3 options were (i) population treatment in years $1-5$, (ii) child-targeted treatment in years $1-5$, and (iii) population treatment in year 1 , and child-targeted treatment in years $2-5$.

The second scenario was a comparison of different frequencies of treatment and different coverages of a 5-year child-targeted treatment programme in a high transmission area. The different frequencies of treatment were (i) every 6 months, (ii) every year, and (iii) every 2 years, representing a total of 10,5 and 3 treatment cycles respectively over the 5-year treatment period. Three levels of coverage were investigated; (i) $50 \%$, (ii) $70 \%$ and (iii) $90 \%$ of children.

\section{Cost analysis}

The cost data used in the analysis were based on actual expenditure of a control programme on the island of Montserrat (BUNDY et al., 1990). All costs are represented in US\$ at 1988 prices. Details of the cost data have already been given (GUYATT et al., 1993), and all unit costs, except transport costs, remain identical to those presented previously. The transport costs have been changed slightly for 2 reasons. Firstly, a 5\% discount rate was used in annualizing the capital cost of the vehicle and, secondly, only the cost for the amount of time the vehicles were actually used has been attributed to the programme. In this analysis, one treatment cycle was assumed to require the vehicle for only 6 months; therefore the transport costs were half that of the previous analysis for yearly and 2-yearly treatments. The transport costs also differed in child-targeted and population treatment because of the different sizes of the target population. The transport cost for child-targeted treatment was assumed to be half that for population treatment, as only one vehicle was required for child-targeted treatment, and 2 for population treatment. The cost of each delivery programme was assumed to be identical in both the high and low transmission areas.

\section{Effectiveness analysis}

In this study, effectiveness was confined to measures of the reduction in infection and disease prevalence as a result of the treatment programme. These 2 effectiveness measures were expressed as the number of infection cases prevented and the number of disease cases prevented, respectively.

The total effectiveness was estimated as the sum of the effectiveness in children and adults, weighted by the proportions of children and adults in the population. The effectiveness was assessed over a period of 10 years; for the duration of the 5 year treatment programme and for 5 years following it.

\section{Cost-effectiveness analysis}

The use of discounting is essential to cost-effectiveness analysis, although the precise discount rate is controversial. In the present study, both the costs and effectiveness of each programme were discounted using a discount rate of $5 \%$ (DRUMMOND et al., 1987). Using alternative discount rates of $3 \%$ and $10 \%$ did not alter the qualitative results; the general conclusions remain unchanged (data not shown).

The most effective option is that which maximizes the effectiveness measure (e.g. the number of disease cases prevented), while the most cost-effective option is that

Table 2. A comparison of the cost-effectiveness of different treatment scenarios for ascariasis (A, child-targeted; B, population followed by child-targeted; and $C$, population) in low and high transmission areas ${ }^{\mathrm{a}}$

\begin{tabular}{lcccccc}
\hline & \multicolumn{2}{c}{ Low transmission area } & \multicolumn{3}{c}{ High transmission area } \\
& A & B & C & A & B & C \\
\hline Cost ('000, US\$) & 122 & 144 & 225 & 122 & 144 & 225 \\
Effectiveness ('000) & & & & & \\
$\quad$ No. of disease cases prevented & 41 & 43 & 50 & 68 & 75 & 92 \\
$\quad$ No. of infection cases prevented & 109 & 139 & 249 & 66 & 85 & 163 \\
Cost-effectiveness ratio (US\$) & & & & & \\
$\quad$ Cost per disease case prevented & 2.94 & 3.34 & 4.53 & 1.78 & 1.92 & 2.45 \\
$\quad$ Cost per infection case prevented & 1.12 & 1.04 & 0.90 & 1.84 & 1.70 & 1.38 \\
\hline
\end{tabular}

assuming treatment is given every year, when $90 \%$ of children and $50 \%$ of adults are treated. Cost and effectiveness have been rounded up to the nearest thousand, but the cost-effectiveness ratios were calculated from the original data. 
which minimizes the cost-effectiveness ratio (e.g. the cost per disease case prevented). The incremental cost-effectiveness ratio indicates the extra costs required to achieve an extra gain in effectiveness by implementing a more expensive but more effective option.

\section{Results}

\section{Child-targeted and population treatment}

The cost and effectiveness of the 3 options for agetargeted treatment in the low and high transmission areas are given in Table 2. The cheapest option was childtargeted treatment, and the most expensive was population treatment. The cost of population treatment followed by child-targeted treatment lay between these.

In both transmission areas, the most effective option was population treatment, since it prevented the highest number of disease and infection cases. Population treatment followed by child-targeted treatment prevented fewer cases than population treatment, but more cases than child-targeted treatment on its own. In terms of the number of infection cases prevented, the most effective and cost-effective option was population treatment. However, although population treatment prevented the highest number of disease cases, child-targeted treatment cost the least per disease case prevented, and was therefore the most cost-effective option in terms of morbidity reduction. This highlights the problem of using the reduction in infection cases as a measure of disease reduction.

The incremental cost-effectiveness ratio provides information on the extra cost per extra unit gain in effectiveness, for employing the more effective, but more expensive, option. Employing population followed by child-targeted treatment instead of child-targeted treatment alone cost US\$2.90 per extra disease case prevented in the high transmission area, compared with US\$ 12.20 in the low transmission area. Using population treatment alone instead of population followed by child-targeted treatment would have cost US\$ 4.86 per extra disease case prevented in the high transmission area and US\$12.58 in the low transmission area. It is evident that it is less expensive per unit gain in effectiveness to move from child-targeted to population followed by child-targeted treatment than to choose population treatment alone instead of the combined approach. In both cases, the incremental cost-effectiveness ratios are higher

Table 3. A comparison of the cost-effectiveness of different combinations of frequency of treatment and coverage for child-targeted treatment for ascariasis in a high transmission area $^{\mathbf{a}}$

\begin{tabular}{|c|c|c|c|c|}
\hline & & Frequen & $y$ of $t r$ & Eatment \\
\hline & Coverage & $\begin{array}{l}\text { Every } \\
6 \text { months }\end{array}$ & $\begin{array}{l}\text { Every } \\
\text { year }\end{array}$ & $\begin{array}{l}\text { Every } \\
2 \text { years }\end{array}$ \\
\hline Cost ('000, US\$) & $\begin{array}{l}0.5 \\
0.7 \\
0.9\end{array}$ & $\begin{array}{l}145 \\
194 \\
243\end{array}$ & $\begin{array}{r}72 \\
97 \\
122\end{array}$ & $\begin{array}{l}44 \\
58 \\
73\end{array}$ \\
\hline $\begin{array}{l}\text { No. of disease } \\
\text { cases prevented }\left({ }^{\prime} 000\right)\end{array}$ & $\begin{array}{l}0.5 \\
0.7 \\
0.9\end{array}$ & $\begin{array}{l}52 \\
70 \\
83\end{array}$ & $\begin{array}{l}32 \\
50 \\
68\end{array}$ & $\begin{array}{l}20 \\
33 \\
49\end{array}$ \\
\hline $\begin{array}{l}\text { No. of infection } \\
\text { cases prevented ('000) }\end{array}$ & $\begin{array}{l}0.5 \\
0.7 \\
0.9\end{array}$ & $\begin{array}{r}45 \\
75 \\
111\end{array}$ & $\begin{array}{l}23 \\
40 \\
66\end{array}$ & $\begin{array}{l}13 \\
23 \\
39\end{array}$ \\
\hline $\begin{array}{l}\text { Cost per disease } \\
\text { case prevented (US\$) }\end{array}$ & $\begin{array}{l}0.5 \\
0.7 \\
0.9\end{array}$ & $\begin{array}{l}2 \cdot 80 \\
2 \cdot 77 \\
2 \cdot 92\end{array}$ & $\begin{array}{l}2.25 \\
1.95 \\
1.78\end{array}$ & $\begin{array}{l}2.16 \\
1.78 \\
1.48\end{array}$ \\
\hline $\begin{array}{l}\text { Cost per infection } \\
\text { case prevented (US\$) }\end{array}$ & $\begin{array}{l}0.5 \\
0.7 \\
0.9\end{array}$ & $\begin{array}{l}3 \cdot 24 \\
2 \cdot 59 \\
2 \cdot 19\end{array}$ & $\begin{array}{l}3 \cdot 13 \\
2 \cdot 41 \\
1 \cdot 84\end{array}$ & $\begin{array}{l}3 \cdot 29 \\
2.53 \\
1 \cdot 89\end{array}$ \\
\hline
\end{tabular}

${ }^{2}$ Cost and effectiveness have been rounded up to the nearest thousand, but the cost-effectiveness ratios were calculated from the original data. in the low transmission area, implying that implementation of these more expensive options would be more favourable in the high transmission area.

\section{Coverage and frequency of treatment}

The cost and effectiveness of the different combinations of frequency and coverage of child-targeted treatment in a high transmission area are given in Table 3. The most effective option, for both infection and disease case prevention, was to treat most people very frequently (i.e. $90 \%$ of children every 6 months). This was also, however, the most expensive option. The cheapest option was to treat few people less frequently (i.e. $50 \%$ of children every 2 years), but this was also the least effective option.

The most cost-effective option in terms of disease cases prevented was treatment every 2 years at a high coverage rate $(90 \%$ of children). For infection case prevention, the most cost-effective option was treatment of $90 \%$ of children every year. Here again, measuring effectiveness in terms of infection cases prevented can be misleading if the aim of control is to reduce disease.

The incremental cost-effectiveness of increasing the frequency of treatment at a given coverage, or increasing the coverage at a given frequency of treatment, can also be important in deciding whether to invest in more frequent treatment or in enhancing the proportion of the population treated.

Considering first the effect of increasing the frequency of treatment at any given coverage, the analysis demonstrated that increasing the frequency from every 2 years to every year cost an extra US\$ $2.28-2.55$ per extra disease case prevented, depending on the coverage. Increasing the frequency of treatment from every year to every 6 months required a much higher investment per extra disease case prevented. It cost between US\$ 3.72 and US\$ 8.23 per extra disease case prevented, the cost increasing with the coverage. This suggests that, whereas increasing the frequency of treatment to every year rather than every 2 years may be a reasonable option, increasing the frequency from every year to every 6 months may not be a good investment since the cost per extra disease case prevented is high, particularly at high coverage (Fig. 1).

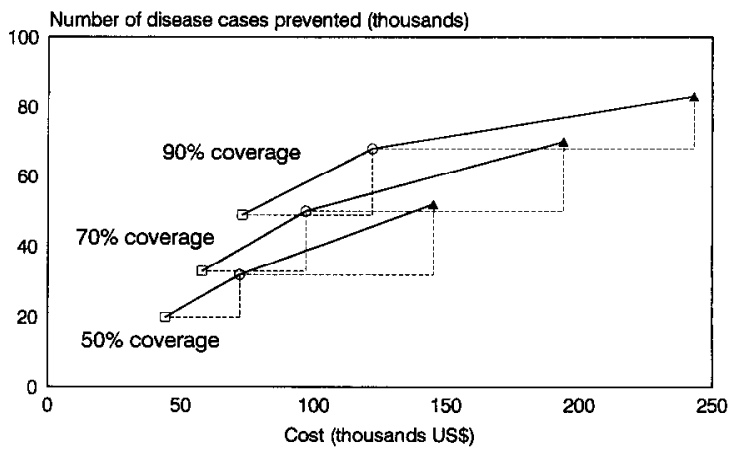

Fig. 1. The relationship between the cost and the number of disease cases prevented when increasing the frequency of child-targeted treatment given 3 levels of coverage: treatment every 2 years $(\square)$, yearly $(O)$ and every 6 months $(\Delta)$. The dashed lines illustrate the extra cost and extra effectiveness of treating more frequently, and the solid line represents the incremental cost-effectiveness ratio.

For any given frequency of treatment, higher coverage resulted in more disease cases prevented, but at additional cost. The cost per extra disease case prevented by treating more children varied with the frequency of treatment. The more frequent the treatments, the greater the investment required for every extra disease case prevented by treating a higher proportion of the children. For instance, increasing the coverage from $50 \%$ to $70 \%$ would cost US\$2.67 per extra disease case 
Number of disease cases prevented (thousands)

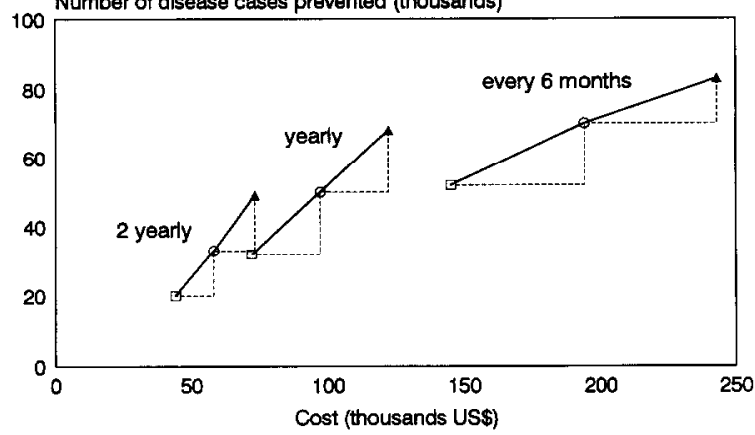

Fig. 2. The relationship between the cost and the number of disease cases prevented when increasing the coverage of child-targeted treatment given different frequencies of treatment: $50 \%(\square), 70 \%(O)$ and $90 \%(\Delta)$ coverage. The dashed lines illustrate the extra cost and extra effectiveness of treating more children, and the solid line represents the incremental cost-effectiveness ratio. (These are the same data points as those in Fig. 1, but connected by different lines.)

prevented if treatment were administered every 6 months, compared to US\$ 1.17 with 2-yearly treatment cycles (Fig. 2). The lines are steeper in Fig. 2 than in Fig. 1, indicating more cost-effective increments.

\section{Discussion}

With the exception of drug efficacy, the coverage and frequency of treatment are often the only aspects of a delivery programme that can be controlled. This analysis, therefore, addressed 2 central issues in control design. Drug efficacy, which can be controlled through the choice of drug and dosage, will be the subject of a separate analysis.

This study investigated the issues of target group, coverage and frequency of treatment in the control of $A$. lumbricoides. Since the analysis was based on costs from an actual control programme, the results are clearly specific to the programme described. The conclusions of the analysis may therefore not be applicable to all mass delivery programmes. Nevertheless, one observation applicable to helminth control in general is that prevalence is a poor indicator of underlying disease patterns, yet it remains the most commonly used measure for assessing control (BUNDY et al., 1992).

The analysis demonstrated that, of the 3 strategies of population and child-targeted treatment, child-targeted treatment alone was the most cost-effective option. The main reason for this was that the intensity of infection was highest in the child age classes, and therefore treating children provided benefit to adults since the rate of transmission was greatly reduced. In this analysis, the unit cost of treating children was assumed to be identical to that for adults. In practice, the costs are likely to be much smaller for children, since they are more easily accessible through schools (WORLD BANK, 1993). Including such a differential cost advantage would further favour child-targeted treatment.

The study investigated the optimal coverage and frequency of treatment by assessing the cost-effectiveness of child-targeted treatment in a high transmission area, given variation in how often and how many children were treated. Previous theoretical studies have investigated a control criterion for chemotherapy, which corresponds to the threshold coverage per time period required to eradicate infection or to control infection to a given level (ANDERSON \& MAY, 1985; ANDERSON \& MEDLEY, 1985). There has been no attempt, however, to dissect the interaction between coverage and frequency of treatment, or to investigate the implications for costeffectiveness. Since the programme was assumed to last for 5 years, it is obvious that the less frequent the treatments, the fewer treatments given. The results from this study predict that it is more cost-effective to extend the range of an existing cycle than to increase the number of cycles. The main reasons for this are that, firstly, it is cheaper and, secondly the dynamics are such that the lower the infection levels achieved the slower the return to equilibrium levels due to reinfection. MEDLEY et al. (1993) showed that increasing the coverage of treatment was increasingly beneficial to the untreated proportion of the population, because of reduced infection rates. These results, however, are dependent on the level of transmission in the host community. Further research is continuing to investigate the relationship between transmission, coverage and frequency of treatment.

The most cost-effective option for child-targeted treatment was shown to be 2-yearly treatment of $90 \%$ of the children. However, it is possible that a 2-yearly treatment programme could be chosen, but only $70 \%$ coverage achieved. In this situation, one may consider trying to increase coverage to $90 \%$. This might involve extra cost to motivate the community (e.g. through health education) and the staff (e.g. through incentives). In this instance, one would be interested in determining the maximum amount of money that could be spent trying to achieve a $90 \%$ coverage, so that this would still be more cost-effective than leaving the coverage at $70 \%$. This can be calculated by determining how much more the costs of the $90 \%$ coverage programme could be increased while still maintaining a lower cost-effectiveness ratio than leaving coverage at $70 \%$. The analyses suggested that, on top of the extra treatment costs, it would be possible to invest up to US\$14967 over the 5-year programme in increasing coverage from $70 \%$ to $90 \%$, and this would still be the more cost-effective option.

In the circumstances described here, enhancing coverage is a more cost-effective approach than increasing frequency. Further research will look at the sensitivity of the results to variability in both the epidemiological and economic frameworks.

\section{Acknowledgements}

H. L. Guyatt is in receipt of a Wellcome Trust Travelling Research Fellowship. G. F. Medley is a Royal Society University Research Fellow. D. A. P. Bundy thanks the Wellcome Trust for financial support. $M$. S. Chan was supported by the Edna McConnell Clark Foundation, as was the work on population dynamics and modelling.

\section{References}

Anderson, R. M. \& May, R. M. (1982). Population dynamics of human helminth infections: control by chemotherapy. $\mathrm{Na}$ ture, 297, 557-563.

Anderson, R. M. \& May, R. M. (1985). Helminth infections of humans: mathematical models, population dynamics and control. Advances in Parasitology, 24, 1-101.

Anderson, R. M. \& Medley, G. F. (1985). Community control of helminth infections of man by mass and selective chemotherapy. Parasitology, 90, 629-660.

Bundy, D. A. P. (1990). Control of intestinal nematode infections by chemotherapy: mass treatment versus diagnostic screening. Transactions of the Royal Society of Tropical Medicine and Hygiene, 84, 622-625.

Bundy, D. A. P., Cooper, E. S., Thompson, D. E., Didier, J. M. \& Simmons, I. (1987). Epidemiology and population dynamics of Ascaris lumbricoides and Trichuris trichiura infection in the same community. Transactions of the Royal Society of Tropical Medicine and Hygiene, 81, 987-993.

Bundy, D. A. P., Wong, M. S., Lewis, L. L. \& Horton, J. (1990). Control of geohelminths by delivery of targeted chemotherapy through schools. Transactions of the Royal Society of Tropical Medicine and Hygiene, 84, 115-120.

Bundy, D. A. P., Hall, A., Medley, G. F. \& Savioli, L. (1992). Evaluating measures to control intestinal parasitic infections. World Health Statistics Ouarterly, 45, 168-179.

Chai, J. Y., Kim, K. S., Hong, S. T., Lee, S. H. \& Seo, B. S. (1985). Prevalence, worm burden and other epidemiological parameters of Ascaris lumbricoides infection in rural communities in Korea. Korean Fournal of Parasitology, 23, 241246.

Chan, M. S., Medley, G. F., Bundy, D. A. P. \& Guyatt, H. L. (in press). The development and validation of an age struc- 
tured model for the evaluation of disease control strategies for intestinal helminths. Parasitology.

Croll, N. A., Anderson, R. M., Gyorkos, T. W. \& Ghadirian, E. (1982). The population biology and control of Ascaris lumbricoides in a rural community in Iran. Transactions of the Royal Society of Tropical Medicine and Hygiene, 76, 187-197.

Drummond, M. F., Stoddard, T. L. \& Torrance, G. W. (1987). Methods for the Economic Evaluation of Health Care Programmes. Oxford: Oxford University Press.

Guyatt, H. L. \& Bundy, D. A. P. (1991). Estimating prevalence of community morbidity due to intestinal helminths: prevalence of infection as an indicator of the prevalence of disease. Transactions of the Royal Society of Tropical Medicine and Hygiene, 85, 778-782.

Guyatt, H. L., Bundy, D. A. P., Medley, G. F. \& Grenfell, B. T. (1990). The relationship between the frequency distribution of Ascaris lumbricoides and the prevalence and intensity of infection in human communities. Parasitology, 101, 139-143.

Guyatt, H. L., Bundy, D. A. P. \& Evans, D. (1993). A population dynamic approach to the cost-effectiveness analysis of mass anthelmintic treatment: effects of treatment frequency on Ascaris infection. Transactions of the Royal Society of Tropical Medicine and Hygiene, 87, 570-575.

Medley, G. F., Guyatt, H. L. \& Bundy, D. A. P. (1993). A quantitative framework for evaluating the effect of community treatment on the morbidity due to ascariasis. Parasito$\log y, 106,211-221$.

Muller, R. (1975). Worms and Disease: a Manual of Medical Helminthology. Lundon: William Heinemann Medical Books.

Prescott, N. M. (1987). The economics of schistosomiasis chemotherapy. Parasitology Today, 3, 21-24.

Savioli, L., Bundy, D. \& Tomkins, A. (1992). Intestinal parasitic infections: a soluble public health problem. Transactions of the Royal Society of Tropical Medicine and Hygiene, 86, 353354.

Warren, K. S., Bundy, D. A. P., Anderson, R. M., Davis, A. R., Henderson, D., Jamison, D. T., Prescott, N. \& Senft, A. (1993). Helminth infections. In: Disease Control Priorities in Developing Countries, Jamison, D. T., Mosley, W. H., Measham, A. R. \& Bobadilla, A. L. (editors). Oxford: Oxford University Press, pp. 131-160.

World Bank (1993). World Development Board: Investing in Health. Oxford: Oxford University Press.

Received 14 March 1994; revised 12 May 1994; accepted for publication 22 fuly 1994

\section{Announcement}

\section{ROYAL SOCIETY OF TROPICAL MEDICINE AND HYGIENE}

The Transactions of the Royal Society of Tropical Medicine and Hygiene is issued bi-monthly (six parts per year) and publishes not only papers presented at Society meetings and symposia but also papers and correspondence submitted from all over the world on every aspect of tropical medicine and international health. Supplements on specialist topics are published from time to time.

The Transactions is available to Libraries and non-Fellows at an annual subscription of $£ 120.00$. It is dispatched by accelerated surface post at no extra charge.

The annual subscription for Fellows is $£ 50.00$. Applications for Fellowship must be made on the correct form, obtainable from the address below. The rate for bona fide students is £25.00.

Further information and application forms for Fellowship can be obtained from:

The Honorary Secretaries Royal Society of Tropical Medicine and Hygiene Manson House 26 Portland Place London, W1N 4EY, UK Telephone: +44(0) 1715802127 Fax: +44(0) 1714361389
Subscription orders from libraries and non-Fellows should be sent to:

Royal Society of Tropical Medicine and Hygiene Subscription Department

Media House

Eccleston Road

Maidstone, ME15 6QN, UK

Telephone: +44 (0) 1622756668

Fax: +44 (0) 1622679774 